\title{
Topical gel based nanoparticles for the controlled release of oleanolic acid: design and in vivo characterization of a cubic liquid crystalline anti-inflammatory drug
}

\author{
Zhiqi Shi ${ }^{* *}$, Shugang Pan ${ }^{2,3}$, Luolin Wang ${ }^{4}$ and Sha Li ${ }^{5^{*}}$
}

\begin{abstract}
Background: Oleanolic acid (OA) has multiple pharmaceutical applications including anti-inflammatory activity, but low permeability of the molecule limits its widespread use.

Methods: A cubic liquid crystalline nanoparticle (LCNP)-based gel was prepared as a potential topical delivery system for OA. The LCNP-based gel was optimized using rheological, drug release kinetic, and ex vivo permeation studies.

Results: The studies showed that the OA was trapped in the interior of the LCNP with a crystal form of Pn3m space. The optimized LCNP formulation performed well using in vitro release studies for up to $12 \mathrm{~h}(85.49 \pm 0.21 \%)$. Ex vivo permeation studies showed that the LCNP-based gel formulation was superior to a standard gel formulation. The $\mathrm{r}^{2}$ value from the Peppas equation indicated good linearity, but showed irregular (non-Fickian) diffusion, suggesting that drug release was controlled by multiple processes.

Conclusions: In this study, OA-loaded LCNPs were prepared by the precursor method, resulting in a wellcharacterized OA-LCNP gel preparation. The gel was shown to be effective in a rodent carrageenan-induced hind paw inflammation model with sustained efficacy after a single application.
\end{abstract}

Keywords: LCNP-based gel, Oleanolic acid, Anti-inflammatory, Permeation studies

\section{Introduction}

Many plants in nature have been proven to have a variety of pharmacological activities, prompting scientists to find ingredients with specific activities in them for better treatment of various diseases. Many triterpenoids including OA have been shown to have a wide range of pharmacological activities [1] including being an antioxidant, hypoglycemic, anti-inflammatory, antibacterial, and

\footnotetext{
* Correspondence: shi_mars@foxmail.com; s10426@foxmail.com

'Changzhou Institute of Industry and Technology, No.28\#, Mingxin Road, Changzhou 213164, Jiangsu Province, China

${ }^{5}$ Wuxi Hospital of Traditional Chinese Medicine, No.8\#, Zhongnan Road, Wuxi 214071, Jiangsu Province, China

Full list of author information is available at the end of the article
}

hepatoprotective [2-8]. The findings of the referenced study revealed OA to possess dose related antiinflammatory activity in a variety of test models including carrageenan-induced oedema in rats [5]. It has been reported that OA could inhibits mouse paw edema and effectively increase the number of Treg cells and the expression of IL-10 and IL-35 $[9,10]$. In addition, OA also caused a pleiotropic antibacterial effect as they were able to influence various cellular functions including induction of the stress response [11]. It may be concluded that OA, whose spectrum of anti-inflammatory activity appears to be different from classical NSAIDs, would be of therapeutic value $[12,13]$. 
However, OA is a class IV drug according to the Biopharmaceutics Classification System, and has an absolute oral bioavailability of only $0.7 \%$ because of its low permeability (Papp $=1.1-1.3 \times 10^{-6} \mathrm{~cm} / \mathrm{s}$ in the apical to basolateral direction at 10 and $20 \mu \mathrm{M}$ ) and low aqueous solubility $(<1 \mu \mathrm{g} / \mathrm{mL})[14,15]$. Several approaches including recrystallization [16], solid dispersion [17], nanoemulsions [18], and drug-phospholipid complex techniques [19] have been used to attempt to improve the bioavailability of OA.

Cubic liquid crystal nanoparticles (LCNP) are now being investigated as a universal carrier for drug delivery [20-24] to improve the oral bioavailability of the drug because of high encapsulation efficiency, drug loading and bio-affinity [25]. In our previous study, we prepared curcumin and piperine-loaded LCNP using a precursor injection method. The results showed that the oral bioavailability of LCNP-curcumin was significantly improved [26].

Based on our previous work and referenced evidence, in this study we explored the possibility of LCNP as a carrier system for a topical formulation of OA. In addition, in this study we also evaluated the in vitro permeability of the drug through rat skin and determined the anti-inflammatory activity of the LCNP gel.

\section{Materials and methods \\ Material}

Phytantriol (purity > 98\%) was purchased from TCI (Tokyo, Japan). OA was acquired from Acetar Biotech Inc. (Shanxi, China). Lutrol F127, propylene glycol, glycerin, carbopol 934 (CP), HPMC K4M, ethanol, were all acquired from Shanhe Pharmaceutical Accessories Co., Ltd. (Anhui, China). Sodium dodecyl sulfate was purchased from Hunan Er-kang Pharmaceutical Co., Ltd. (Hunan, China). Azone was purchased from Tianmen scientific Pharmaceutical Co., Ltd. (Hubei, China). Triethanolamine was given as a gift from Jiangxi $\alpha$-Hitech scientific Pharmaceutical Co., Ltd. (Jiangxi, China). Ultrapure water was produced by using a Milli-Q purification system from Millipore (Massachusetts, USA). Acetonitrile and methanol (both HPLC grade) were purchased from Merck (New Jersey, Germany). Standards of OA (CAS: 508-02-1, > 95\%) were purchased from Nation Institutes for Food and Drug Control (Beijing, China). All materials were used as received.

\section{Animals}

Male rats (weighing 180-220 g) were obtained from Guangdong Medical Laboratory Animal Center, and were housed under $12 \mathrm{~h}: 12 \mathrm{~h}$ light-dark cycle, constant temperature of $22 \pm 2{ }^{\circ} \mathrm{C}$ and $40-50 \%$ humidity. All laboratory animals had free access to water and food. The study was carried out in compliance with the ARRIVE guidelines. The experimental protocols and treatments were all approved by Ethics Committee of Guangdong Provincial Institute of Traditional Chinese Medicine.

\section{Methods}

\section{Screening of excipients}

When developing the LCNP system, suitable liquid, lipid and surfactants were selected for use, and were generally considered safe (GRAS). At present, the excipients used to prepare LCNP mainly include phytantriol and glycerol monooleate(GMO). These two materials have different ternary phase diagram regions, and the conditions for forming cubic liquid crystals are also different (Fig. 1) $[32,33]$.

OA and excipient (Phytantriol or GMO) were weighed and dissolved in $2.5 \mathrm{~mL}$ of absolute ethanol simultaneously with sonication at $80 \%$ of maximum power for $30 \mathrm{~min}$. The organic phase was added dropwise to the aqueous phase $20 \mathrm{~mL}$ containing stabilizer F127 under agitation at $500 \mathrm{rpm}$ for $3 \mathrm{~h}$ to form a crude dispersion, which was then sonicated (Misonix XL2000, Misonix Inc., Farmingdale, N.Y.) in a pulsed mode $(0.5 \mathrm{~s}$ pulse interrupted by $0.5 \mathrm{~s}$ interrupt) at $40 \%$ of maximum power for $10 \mathrm{~min}$ to produce a milky dispersion and to reduce particle size.

\section{Surfactant screening}

In order to prepare stable LCNPs, two surfactants (Lutrol F127 and Vitamin E Acetate) were screened.

\section{Preparation of OA loaded LCNP}

The nanoparticles were prepared using the precursor method described in our previous study [26, 27]. Drugs and excipients were weighed according to the prescriptions in Table 1. The phytantriol and OA were dissolved in $2.5 \mathrm{~mL}$ of absolute ethanol simultaneously with sonication at $80 \%$ of maximum power for $30 \mathrm{~min}$. The organic phase was added dropwise to the aqueous phase $(20 \mathrm{~mL})$ containing stabilizer F127 under agitation to form a crude dispersion, which was then sonicated in a pulsed mode $(0.5 \mathrm{~s}$ pulse interrupted by $0.5 \mathrm{~s}$ interrupt $)$ at $40 \%$ of maximum power for $10 \mathrm{~min}$ to produce a milky dispersion and to reduce particle size. In addition, LCNP containing OA with loading amounts of 6,8 and $10 \%$, respectively, were prepared and their effects on encapsulation efficiency were investigated. The effects of different stirring speeds $(500,1000,1500 \mathrm{rpm})$ and homogenization cycle $(3,6,9$ cycles) were optimized based on the measurements of particle size and encapsulation efficiency, respectively. The OA-loaded LCNP 

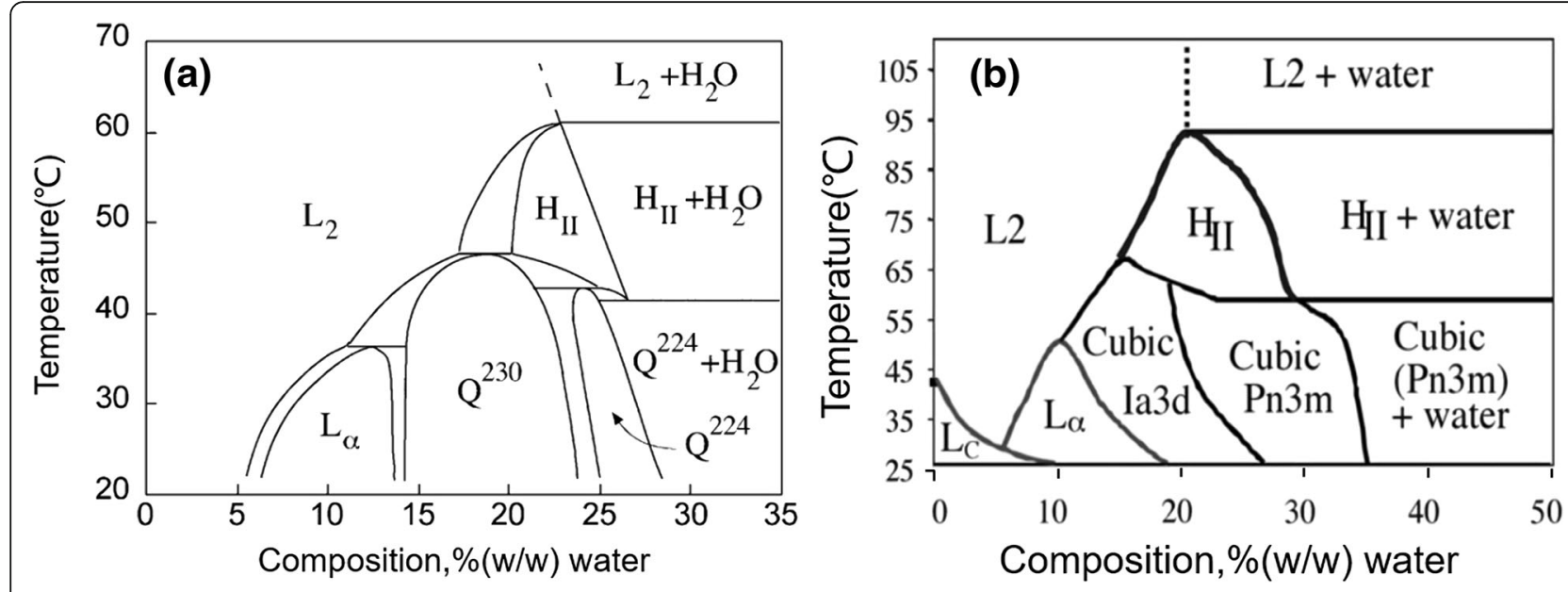

Fig. 1 Binary phase diagram of phytantriol-water (a) and glycerol monooleate-water (b)

dispersion obtained was freeze-dried to be a dry and free-flowing powder.

\section{Particle size, micrographs and crystal cell parameter measurements of OA-loaded LCNP}

The size of LCNP was measured using dynamic light scattering (Nano patica SZ100, H field, Iwate Prefecture, Japan); we used an average of five measurements. Micrograghs of the nanoparticles were investigated by scanning electron microscopy (SEM; 1430VP, Leo, Sauerlach, Germany) [21]. In determining the size of the nanoparticles, the cubic phase nanoparticle suspension was first placed on silicon water through a capillary tube, then allowed to dry at room temperature. Thereafter, they were coated with platinum for $2 \mathrm{~min}$ in the ion sputtering instrument. The coated samples were measured at an acceleration voltage of $15 \mathrm{kV}$. Average particle size and polydispersity index were measured by photon correlation spectroscopy at a $90^{\circ}$ angle at $25^{\circ} \mathrm{C}$.

Polarizing light microscopy (BX41P, Olympus, Tokyo, Japan) with a (MATS-U55S, Tokaihit, Shizuoka, Japan) heater set between a range of $20-60{ }^{\circ} \mathrm{C}$ [21] was used in this study The crystal cell parameter measurement used a Bruker Nanostar small-angle X-ray scattering (SAXS) camera with pinhole collimation for point focus geometry. The sample was placed

Table 1 Different Formulations for OA-loaded LCNP

\begin{tabular}{lllll}
\hline S.No. & $\begin{array}{l}\text { Formulations } \\
\text { parameters }\end{array}$ & \multicolumn{3}{l}{ Formulations } \\
\cline { 3 - 5 } & OAF1 & OAF2 & OAF3 \\
\hline 1 & Phytantriol $(\mathrm{mg})$ & 70 & 80 & 90 \\
2 & F127 $(\mathrm{mg})$ & 30 & 20 & 10 \\
3 & OA $(\mathrm{mg})$ & 6 & 8 & 10 \\
\hline
\end{tabular}

in the quartz capillary tube, and the optics and sample chamber were placed under vacuum to minimize air scatter. The results were normalized for sample transmission after the background was subtracted and integrated using Bruker AXS software. The diffraction patterns obtained were converted to plots of intensity versus $q$ value.

\section{Zeta potential analysis of OA-loaded LCNP}

The nanoparticle surface charge was measured by determining the zeta potential using a Zetasizer (3000HS Malvern Instruments, UK). The LCNP suspension was diluted with double distilled water (1: $100, \mathrm{w} / \mathrm{w})$ to obtain a uniform dispersion before testing, and the conductivity was then measured at $25^{\circ} \mathrm{C}$ [28]. All values calculated were the average of three independent measurements (mean \pm standard deviation).

\section{Drug entrapment efficiency (DEE) and drug loading (DL) of OA-loaded LCNP}

Nanoparticles were embedded in a Sephadex G-50-filled microcolumn under a centrifugal force of $500 \mathrm{rpm}$ for 5 min and then eluted with the same volume of ultrapure water. The eluate was obtained by centrifugation at 2000 $\mathrm{rpm}$ for $5 \mathrm{~min}$. After repeating the same procedure for five times, the eluate was then collected and dissolved in methanol. The drug content of the eluate was measured in triplicate by a validated HPLC method. DEE of LCNP was calculated as follows: DEE $(\%, w / w)=$ Total amount of drug- Amount of drug in supernatant $\times 100 /$ Total amount of drug. DL $(\%, w / w)=$ Initial drug - free drug/Mixed lipid $\times 100$. 


\section{In vitro release from OA-loaded LCNP}

The in vitro release was measured by a dialysis method. Briefly, the nanoparticles and the suspensions were placed in the dialysis bag (Biosharp, Hefei, China) with a molecular cut off of $14 \mathrm{kDa}$, and the dialysis bag was suspended in $20 \mathrm{~mL}$ of release medium (containing 0.5\% sodium lauryl sulfate) at $37^{\circ} \mathrm{C}$ while the speed of the magnetic agitation was set at $300 \mathrm{rpm} .100 \mu \mathrm{L}$ of sample was withdrawn at predetermined time interval and replaced with fresh medium. The sample content was then determined by the in vitro HPLC method. The HPLC apparatus used for the quantitation consisted of an Agilent 1200 HPLC system (Agilent, USA) with DAD detector. Compounds were separated on a Kromasil RPC18 column $(4.6 \mathrm{~mm} \times 250 \mathrm{~mm}$, Akzo Nobel, Stockholm, Sweden) using a flow rate of $1.0 \mathrm{~mL} / \mathrm{min}$ with a fixed mobile phase [acetonitrile and $0.1 \mathrm{moL} \cdot \mathrm{L}^{-1}$ ammonium acetate (55:45)]. The wavelength was set at $210 \mathrm{~nm}$. The injection volume was $10 \mu \mathrm{L}$ and the retention time of OA was determined to be $27.665 \mathrm{~min}$. The LCNP matrix contained the same amount of ingredients but without OA, and this was used as a blank for comparison. Calibration curves plotted for OA showed good linearity, with a correlation coefficient $\left(\mathrm{r}^{2}\right)$ of 0.9993 in the concentration ranges tested $\left(10.42 \sim 104.2 \mu \mathrm{g} \cdot \mathrm{mL}^{-1}\right)$.

This analytical method has been validated in accordance with the guidelines of the International Conference on Harmonization of Technical Requirements for Registration of Pharmaceuticals for Human Use (ICH). Parameters such as accuracy, reproducibility, and stability were verified separately. The relative standard deviations (RSD) of intra and inter day analyses were $<3 \%$, and the accuracy of the method was verified with the average recovery rates of $99.02 \%$.

\section{Preparation of OA-LCNP-based gel}

After optimizing the OA-LCNP method, the topical gel loaded with OA-LCNP was prepared by a dispersion swelling technique using Carbopol 934 and HPMC K4M. All excipients were weighed as shown in Table 2, and then Carbopol 934 and HPMC K4M were simultaneously dispersed in water and allowed to stand for $4 \mathrm{~h}$ to properly swell the polymer. OA-LCNP powder (equivalent to $0.5 \mathrm{mg} / \mathrm{g}$ ) was then added to the polymer gel under constant one-way agitation to avoid air bubbles. Triethanolamine was then added to the gel mixture for crosslinking between Carbopol 934 polymer and HPMC K4M to form a gel. Then, propylene glycol and glycerin were added to the gel to balance its viscosity, azone was added to increase the permeability of the gel, and finally the $\mathrm{pH}$ was adjusted to a skin $\mathrm{pH}$ of $7.4 \pm 0.1$ by addition of $0.1 \mathrm{~N} \mathrm{NaOH}$ [29]. In addition, a conventional OA-loaded gel (NG) was prepared in the same manner without OA-loaded LCNP.

\section{Physical appearance and formulation $\mathrm{pH}$}

Basic physicochemical parameters were screened for different formulations. The color, transparency, homogeneity and appearance of the prepared gel were visually inspected. The $\mathrm{pH}$ of the $1 \%$ aqueous solution of the gel was measured by a pH meter (Mettler Toledo, Switzerland). One gram per meter of gel was dissolved in $100 \mathrm{~mL}$ of distilled water and stored for $2 \mathrm{~h}$ [23]. The $\mathrm{pH}$ of each formulation was measured in triplicate and the average was calculated.

\section{Viscosity and Spreadability analysis of OA-LCNP-loaded topical gel}

In the rheological properties test, all developed formulations were placed in a beaker, placed in the rotor, and then rotated at $10 \mathrm{rpm}\left(25-27^{\circ} \mathrm{C}\right)$ in a Brookfield viscometer (V-550, Thermo). To measure the spreadability of the formulated OA-LCNP gel, we introduced $1 \mathrm{~g}$ of gel per gram between two slides, held the pre-weighed plate over the gel and gradually added more weight until the gel stopped spreading. The final cumulative weight and total time required for gel diffusion were measured and recorded separately. The spreadability of the gel was then calculated according to the formula, i.e., the total weight applied and the gel mass were compared by the time [29]. Spreadibility $=$ Mass $\times$ Length $/$ Time.

\section{Ex vivo drug permeation studies of OA-LCNP-loaded topical gel}

The measurements of drug permeation were performed using a Franz diffusion cell to evaluate the OA release

Table 2 Formulation table for OA-LCNP-based Gel

\begin{tabular}{llllll}
\hline S.No. & Ingredients & OA-LCNPG1 (w/w) & OA-LCNPG2(w/w) & OA-LCNPG3(w/w) & NG(w/w) \\
\hline 1 & CP 934 & $1.5 \%$ & $1.0 \%$ & $0.5 \%$ & $1.0 \%$ \\
2 & HPMC K4M & $0.5 \%$ & $1.0 \%$ & $1.5 \%$ & $1.0 \%$ \\
3 & glycerol & $10 \%$ & $10 \%$ & $10 \%$ & $10 \%$ \\
4 & propylene glycol & $5 \%$ & $5 \%$ & $5 \%$ & $5 \%$ \\
5 & Triethanaolamine & $2 \%$ & $2 \%$ & $2 \%$ & $1 \%$ \\
7 & Azone & $1 \%$ & $1 \%$ & $1 \%$ & $2 \%$ \\
\hline
\end{tabular}


profile from each formulation. The surface area of the release membrane was $1.971 \mathrm{~cm}^{2}$, and the receiving chamber volume was $18.54 \mathrm{~mL}$. Before performing the permeability study, the abdominal skin of the rat was obtained and processed. The subcutaneous tissue was carefully removed by surgery, and the dermal side was wiped with isopropyl alcohol to remove the attached fat layer. The skin was cleaned again with distilled water and then stored at $-18{ }^{\circ} \mathrm{C}$. During the permeability test, the skin was placed between the donor and recipient compartments of the Franz cells with the stratum corneum side facing the donor compartment and the dermis side facing the receptor compartment [30].

One gram (equivalent to $0.5 \mathrm{mg} \mathrm{OA}$ ) of gel (NG, OA-LCNP G1, OA-LCNP G2, OA-LCNP G3, and NG) was added in to the donor compartment. The receptor compartment was filled with PBS ( $\mathrm{pH} 7.4$ ) (containing $0.5 \%$ sodium lauryl sulfate) and kept in full contact with the dermis layer, the temperature was controlled at $37^{\circ} \mathrm{C}$, and the speed of magnetic stirring was performed at $400 \mathrm{rpm} .2 \mathrm{~mL}$ of the receptor solution was withdrawn at an interval of $0.5,1,2$, $3,6,9,12 \mathrm{~h}$. After each sampling, an equal volume of PBS was simultaneously added to the receptor compartment to maintain volume. Each sample was filtered through a $0.45 \mu \mathrm{m}$ polyamide membrane filter (Satorius, Germany), and then the OA content was determined by HPLC. The concentration in the sample taken and the percentage of drug release of the preparations were calculated.

\section{Analysis of permeability OA-LCNP-loaded topical gel}

For each formulation, the percentage of drug permeated through the skin $\left(\mu \mathrm{g} \cdot \mathrm{cm}^{-2}\right)$ was plotted as a function of time. By dividing the slope of the linear portion of the graph by the diffusion cell area $\left(\mu \mathrm{g} \cdot \mathrm{cm}^{-2} \cdot \mathrm{h}^{-1}\right)$, the drug flux (permeability) at steady state (Jss) was calculated. The permeability coefficient (Kp) was calculated by dividing Jss by the initial concentration of the drug in the donor cell $\left(\mathrm{cm} \cdot \mathrm{h}^{-1}\right)$. The enhancement rate (Er) was calculated by dividing the Jss of each formulation by the Jss of the control formulation [30].

\section{Drug release kinetics studies OA-LCNP-loaded topical gel}

The data obtained from in vitro drug release studies were plotted as percent drug release versus time (Zeroorder equation), logarithm of drug residue versus time (First-order equation), percentage of drug release versus square root of time (Higuchi model equation), and the logarithmic relationship between logarithmic drug release and logarithmic time (Korsmeyer equation) to assess drug release mechanisms. The standard values for the release mechanism are listed in Table 5 .

\section{In vivo anti-inflammatory studies of OA-LCNP-loaded topical gel}

Anti-inflammatory studies were performed in adult male Wistar rats weighing $150-250 \mathrm{~g}$ according to a previously reported technique [31]. Briefly, rats were randomly divided into three groups, One experimental group and two control groups ( $n=6$ per group). Group I received topical saline application (control group), Group II received commercially available mometasone furoate gel (standard group), and Group III received LCNP-based gel formulation (test group).

The left hind paw of the rat was first marked directly above the tibia-tar junction. The paw was immersed in the electrolyte column every time until the marker was fixed in order to measure the volume of the constant paw. The test formulation was applied to the left hind paw of the rat for $30 \mathrm{~min}$ before carrageenan-induced inflammation. The initial paw volume (Vo) of the rats was determined just prior to injection of carrageenan, and the volume increase due to fluid excretion was noted from the digital display, and then $0.1 \mathrm{~mL}$ of $1 \%$ carrageenan solution was injected into the saline which located at the subplantar area of the left hind paw of the rat. The paw volume $(\mathrm{Vt})$ was then assessed after 1, 2, 4, 8,12 and $24 \mathrm{~h}$, respectively. The edema rate and inhibition rate of each group are calculated as follows:

$$
\begin{aligned}
& \text { Edema Rate }(\mathrm{E} \%)=(\mathrm{Vt}-\mathrm{Vo}) / \mathrm{Vo} \times 100 \\
& \text { Inhibition Rate }(\mathrm{I} \%)=(\mathrm{Ec}-\mathrm{Et}) / \mathrm{Ec} \times 100
\end{aligned}
$$

Where Ec is the edema rate of control group, and Et is the edema rate of the treated group.

\section{Results and discussion}

\section{Screening of excipients}

It can be seen from Fig. 1 that GMO could form LCNP with different crystal lattices below $60^{\circ} \mathrm{C}$ with water content above $10 \%$, and LCNP can also be formed by phytantriol when the water composition was above $15 \%$ with the temperature below $45^{\circ} \mathrm{C}$. As it was shown in Table 3, we found that higher entrapment efficiency was obtained with Phytantriol $(68.12 \pm 2.36 \%$ w/w) but with larger size particle $(179 \pm 13.63 \mathrm{~nm})$. However, GMO resulted in smaller size particle $(154 \pm 11.36 \mathrm{~nm})$ but with lower entrapment efficiency $(59.41 \pm 2.32 \% \mathrm{w} / \mathrm{w})$ and drug loading $(10.12 \pm 0.43 \% \mathrm{w} / \mathrm{w})$. Thus, phytantriol was selected as the suitable excipient.

\section{Surfactant screening}

From the results in Table 4, smaller size particle can be formed by Vitamin E Acetate but with lower entrapment efficiency $(59.41 \pm 2.32 \% \mathrm{w} / \mathrm{w})$ and drug loading $(10.12 \pm$ $0.43 \% \mathrm{w} / \mathrm{w})$, which might be due to the numbers of 
Table 3 Particle size, Zeta potential, and polydispersity index of OA-LCNP

\begin{tabular}{|c|c|c|c|c|c|c|}
\hline \multirow[t]{2}{*}{ No. } & \multicolumn{3}{|c|}{ Formulations(mg) } & \multirow{2}{*}{$\begin{array}{l}\text { Particle } \\
\text { size } \\
(\mathrm{nm}) \pm S D\end{array}$} & \multirow{2}{*}{$\begin{array}{l}\text { DEE } \\
(\% \mathrm{w} / w) \pm \\
\text { SD }\end{array}$} & \multirow{2}{*}{$\begin{array}{l}\mathrm{DL} \\
(\% \mathrm{w} / \mathrm{w}) \pm \\
\mathrm{SD}\end{array}$} \\
\hline & Phytantriol & GMO & F127 & & & \\
\hline 1 & 90 & / & 10 & $179 \pm 13.63$ & $68.12 \pm 2.36$ & $13.04 \pm 0.56$ \\
\hline 2 & / & 90 & 10 & $154 \pm 11.36$ & $59.41 \pm 2.32$ & $10.12 \pm 0.43$ \\
\hline
\end{tabular}

hydroxyl and the net structure of the stabilisers. F127 was chosen as a favorable stabilizer in the after studies.

\section{Screening of formula technology}

We compared 2 methods to prepare LCNP: the precursor injection method and the solvent emulsion evaporation technique. The precursor-injection method exhibited the smallest particle size $(273 \pm 18.92 \mathrm{~nm})$ and highest entrapment efficiency $(70.12 \pm 2.12 \% \mathrm{w} / \mathrm{w})$ compared to the solvent emulsification evaporation technique, which resulted in a particle size of $543 \pm 32.93 \mathrm{~nm}$ and entrapment efficiency of $45.18 \pm 2.08 \% \mathrm{w} / \mathrm{w}$.

\section{Optimization of process parameters}

Several process parameters were determined prior to preparation of the LCNP. The ratio of phytantriol to F127, surfactant concentration, agitation time, agitation speed and homogenization cycle were optimized over a wide range to enable selection of the optimum formulation. Since the quality and efficacy of cubosomes were significantly affected by the properties and concentrations of the surfactant, the ratio of phytantriol to F127 was optimized with the measurement of particle size and encapsulation efficiency [34]. The ratios of phytantriol to F127 were assumed to be 70:30, 80:20 and 90:10, respectively. The results showed that the ratio of phytantriol to F127 was 80:20 with the smallest particle size $(138 \pm 10.21 \mathrm{~nm})$ and the optimal encapsulation efficiency of the nanoparticles is $72.39 \pm 2.36 \% \mathrm{w} / \mathrm{w}$, as shown in Fig. 2.

The effects of different stirring speeds between 500, 1000, $1500 \mathrm{rpm}$ were screened, and $1000 \mathrm{rpm}$ was chosen because it had the lowest particle size $142 \pm$ $11.83 \mathrm{~nm}$ and the best encapsulation efficiency $71.93 \pm$ $2.93 \%$ w/w as shown in Fig. 1.

In addition, stirring time between $15,30,45$, and 60 min was also screened and it was determined that 30 min was optimal to obtain the lowest particle size. The homogenization cycle was screened based on the measurements of particle size and encapsulation efficiency for 3, 6 and 9 cycles. The results showed that as the number of cycles increased, the particle size first decreased and then increased slightly. This may be due to the fact that as the homogenization cycle increased, the system temperature gradually decreased, resulting in an increase in kinetic energy, thus the particles reassemble during preparation [23]. Six cycles achieved a minimum particle size of $142 \pm 9.98 \mathrm{~nm}$ and an optimal drug retention of $72.17 \pm 2.83 \% \mathrm{w} / \mathrm{w}$ as shown in Fig. 1 .

When assessing evaluated parameters, we developed different formulations to take into account optimization of parameters, i.e. 0, 1 (optimization parameters), and the +1 level was certain as given in Table 1 . The LCNP loaded with OA was successfully prepared by the precursor injection method, and the parameters such as particle size, encapsulation efficiency, zeta potential and drug release in vitro were characterized. The LCNP loaded with $\mathrm{OA}$ showed particle size in the range of $129 \pm 12.11 \mathrm{~nm}$ to $272 \pm 21.83 \mathrm{~nm}$. The minimum particle size was $129 \pm 12.11 \mathrm{~nm}$ of the formulation OAF1, which is the certain condition for local delivery of LCNP as shown in Table 5 .

The particle size of OAF1 and OAF2 decreased as the concentration of the surfactant increased, but we noted an eventual increase. This may be due to an increase in the homogenization cycle and OA content. As the homogenization cycle increased, the kinetic energy of the system increased, causing particle aggregation [29]. The smaller size may help improve penetration of the drug through the biofilm and aid in targeting. These are considered to be important criteria for topical drug delivery systems that use loaded nanoparticles. In general, the particle size of the nanoparticles should be as small as possible to penetrate the skin more easily, especially in the case of LCNP. Under some circumstances, entrapment efficiency can be considered to be less important

Table 4 Particle size, Zeta potential, and polydispersity index of OA-LCNP

\begin{tabular}{|c|c|c|c|c|c|c|}
\hline \multirow[t]{2}{*}{ No. } & \multicolumn{3}{|c|}{ Formulations(mg) } & \multirow{2}{*}{$\begin{array}{l}\text { Particle } \\
\text { size } \\
(\mathrm{nm}) \pm \text { SD }\end{array}$} & \multirow{2}{*}{$\begin{array}{l}\text { DEE } \\
(\% \text { w/w }) \pm \\
\text { SD }\end{array}$} & \multirow{2}{*}{$\begin{array}{l}\text { DL } \\
(\% w / w) \pm \\
\text { SD }\end{array}$} \\
\hline & Phytantriol & F127 & Vitamin E Acetate & & & \\
\hline 1 & 90 & / & 10 & $104 \pm 14.32$ & $4.46 \pm 1.24$ & $1.02 \pm 0.21$ \\
\hline 2 & 90 & 10 & / & $179 \pm 13.63$ & $68.12 \pm 2.36$ & $13.04 \pm 0.56$ \\
\hline
\end{tabular}




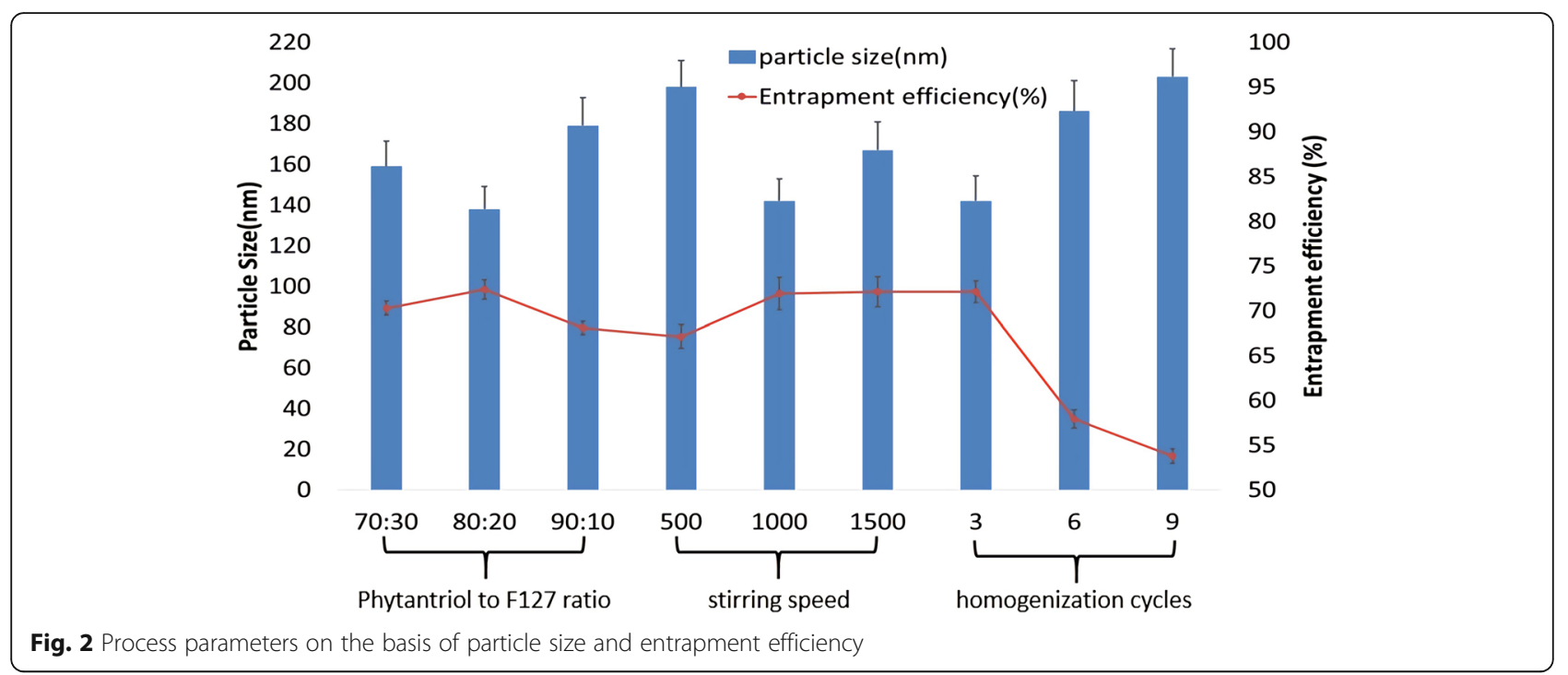

than particle size for LCNP formulation development. The values of zeta potential were in the range of -18.3 to $-21.2 \mathrm{mV}$ for all three formulations. A high zeta potential is related to the storage stability of the nanoparticles, which may indicate that the nanodispersions may not aggregate. We found that the zeta potential increased and then decreased (OAF1 < OAF2).

Determination of the particle size and the distribution of nanoparticles require measurement of the polydispersity index (PDI). A sample having a polydispersity value of less than 0.7 indicates that the nanoparticles are substantially uniformly dispersed. The results showed that the values of the PDI of the three formulations ranged from 0.218 to 0.436 , with the formula CPF2 showing a minimum PDI of 0.218 . The results showed that the PDI value decreased with an increase of phytantriol and then increased. The shape and surface morphology of the LCNP were observed by scanning electron microscope (SEM). The average particle size of the cubic nanoparticles was $152 \mathrm{~nm}$ as determined by dynamic light scattering (DLS) (Fig. 3). The SEM image revealed that the particles were cube-shaped with Pn3m space group and stacked in 3D (Fig. 4).

Due to the optically isotropic properties of LCNP, the cubic liquid crystal appeared dark at $20^{\circ} \mathrm{C}$ in the field of view, which usually indicated the formation of a cubic phase. However, when the sample was heated at $55^{\circ} \mathrm{C}$, the anisotropic image appeared as a fan-like texture, indicating that the Hk phase was formed instead of the cubic phase, which was due to the transformation of the crystal structure (Fig. 5) [35] and also consistent with our previous research [26]. In addition, when the temperature drops to $20^{\circ} \mathrm{C}$, the dark field of the image reappeared which indicated the formation of a cubic phase.

SAXS was used to determine the internal structure of the cubosome nanoparticles. Figure 6 shows a plot of intensity versus the scattering vector and $\mathrm{q}$, obtained from formulations prepared using phytantriol and F127. The OA-loaded LCNP displayed four peaks, where the first two were more intense than the second pair. The relative position of the peaks was in accordance with the bicontinuous cubic phase structure with $\mathrm{Pn} 3 \mathrm{~m}$ space group, indicative of the cubosome nanoparticles with a D-type cubic nanostructure with lattice parameters of $67.4 \AA$.

The encapsulation efficiency of the LCNP loaded with OA was in the range of $68.31 \% \pm 2.86$ to $73.18 \% \pm 3.21$ $\mathrm{w} / \mathrm{w}$. Encapsulation efficiency is related to the crystallinity of the lipid nanoparticles; the more phytantriol in the formulation mixture, the higher with the entrapment efficiency. The drug loading of OA-loaded LCNP was in

Table 5 Particle size, Zeta potential, and polydispersity index of OA-LCNP

\begin{tabular}{lllllll}
\hline Formulations & Drug loaded(\%, w/w) & $\begin{array}{l}\text { Particle size } \\
(\mathbf{n m}) \pm \text { SD }\end{array}$ & Zeta potential & Polydispersity index & DEE(\% w/w) \pm SD & DL(\% w/w) \pm SD \\
\hline OAF1 & 6 & $129 \pm 12.11$ & $-18.3 \mathrm{mV}$ & 0.322 & $73.18 \pm 3.21$ & $12.31 \pm 0.41$ \\
OAF2 & 8 & $159 \pm 17.87$ & $-21.2 \mathrm{mV}$ & 0.218 & $70.93 \pm 3.28$ & $14.12 \pm 0.32$ \\
OAF3 & 10 & $272 \pm 21.83$ & $-19.9 \mathrm{mV}$ & 0.436 & $68.31 \pm 2.86$ & $13.11 \pm 0.74$ \\
\hline
\end{tabular}




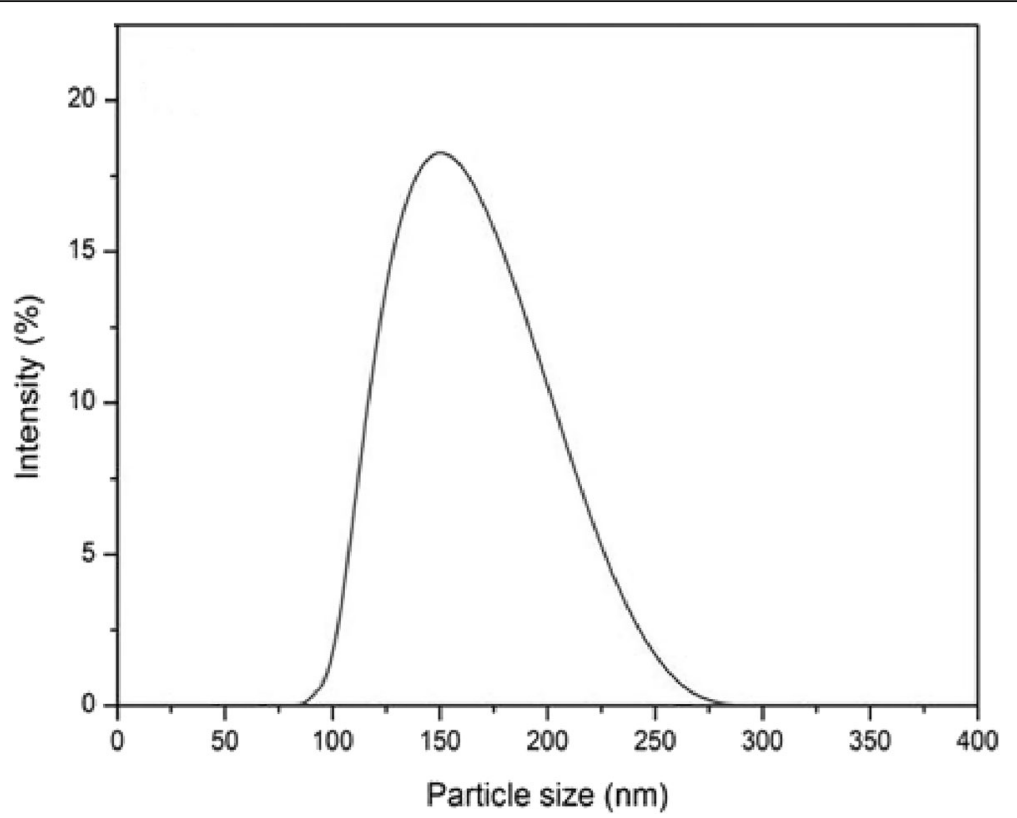

Fig. 3 Particle size distribution of OA-loaded LCNP

the range of $12.31 \% \pm 0.41$ to $14.12 \% \pm 0.32 \mathrm{w} / \mathrm{w}$. Compared with OAF2 $(84.56 \% \pm 0.19)$ and OAF3 $(81.22 \% \pm 0.15)$, OA loading LCNP (OAF1) showed the largest in vitro drug release rate of $85.49 \% \pm 0.21$ within $12 \mathrm{~h}$ as shown in Fig. 7 . The in vitro release of OA from the LCNP dispersion was biphasic with an initial burst effect followed by a gradual release of OA. The initial release may be due to the presence of un-embedded drug in the LCNP dispersion, or it may be due to the presence of dissolved forms of the drug in the outer shell of the liquid lipid and lead to burst in the initial phase. However, release may occur due to corrosion or diffusion of the substrate [28]. The drug provided by the initial burst release immediately produces a rapid therapeutic effect, and the permeability of the drug was improved, while the sustained release of the drug is prolonged and the concentration of the therapeutic agent at the site of action is

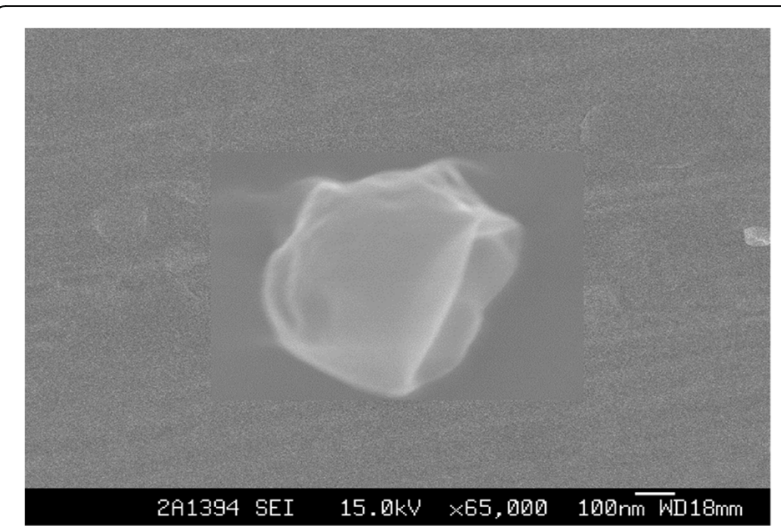

Fig. 4 Morphology of OA-loaded LCNP by scanning electron microscope (Magnification 65,000) maintained. This clearly shows that sustained release can be obtained by using this formulation with a single application.

\section{Characterization of OA-LCNP-loaded topical gel}

The preparations of OA-LCNP-loaded topical gel were lucid, clear, and uniformed in texture, and the $\mathrm{pH}$ ranges of 7.1-7.4 which could easily be tolerated on skin without any stimulation. On account of lowest concentration of HPMC compared with OALCNP-G2 (64,000-71,000 cps) and OA-LCNP-G3 (55,000-63,000 cps), OA-LCNP-G1 had the lowest viscosity (72,000-79,000 cps). OA-LCNP-G1 can be spread easily for application compared to OA-LCNPG2 since its slightly lower viscosity. Results for LCNP-loaded gel characterization are provided in Table 6. Values for percentage in vitro drug release up to $12 \mathrm{~h}$ for OA-LCNP-G1, G2, and G3 are 84.93, 86.78 , and $87.89 \%$, respectively, as given in Fig. 8 .

It was found that the in vitro release of the gel prepared by OA-LCNP was divided into two stages, the initial burst effect followed by the gradual release of OA. The initial burst may be due to the presence of unembedded drug in the LCNP system, or due to the liquid lipid located in the outer shell which contained the lipophilic drug in dissolved form and caused the initial burst. Release may occur through erosion or diffusion of the gel matrix [36]. The initial burst release provided immediate treatment and improved the permeability of the drug. The sustained release could ensure the stable concentration of the drug and maintain for a longer period of time. 
(a)

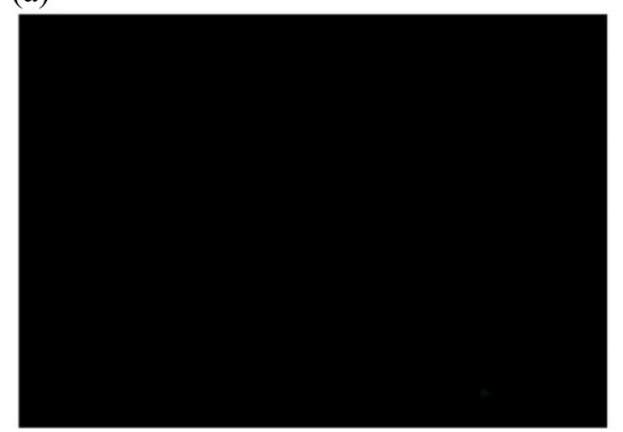

(b)

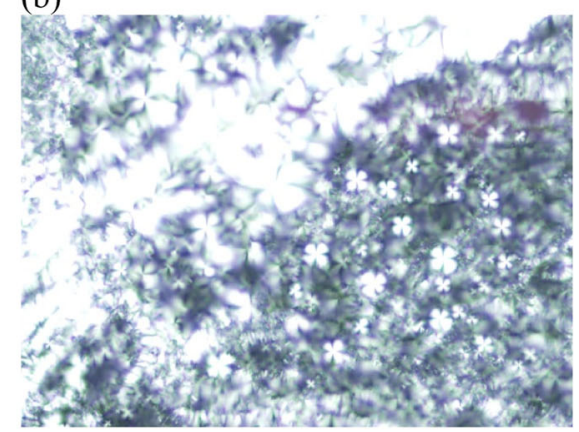

Fig. 5 a Polarizing light micrograph of OA-loaded LCNP at $20^{\circ} \mathrm{C}$ and $\mathbf{b}$ the cubosome at $55^{\circ} \mathrm{C}$

The permeation study suggested that permeability parameters such as steady-state flux (Jss), permeability coefficient $(\mathrm{Kp})$, and increased ratio were significantly higher in both of the OA-LCNP-G1 and OA-LCNP-G2 formulations, compared to OA-LCNP-G3. The cumulative amount of the permeated drug at the end of $12 \mathrm{~h}$ was $371.5,384.7$, and $429.8 \mu \mathrm{g} / \mathrm{cm}^{2}$ with a steady state flux (Jss) of $188.5,195.2$, and $218.0 \mu \mathrm{g} / \mathrm{cm}^{2} / \mathrm{h}$ for OALCNP-G1, OA-LCNP-G2, and OA-LCNP-G3, respectively, as shown in Fig. 9. Value of permeability coefficient is high for OA-LCNP-G1 $(1.16 \mathrm{~cm} / \mathrm{h})>$ OA-LCNPG3 $0.96 \mathrm{~cm} / \mathrm{h})>$ OA-LCNP-G2 $(0.98 \mathrm{~cm} / \mathrm{h})$. There was an increased ratio of OA-LCNP-G3 and OA-LCNP-G2, 1.16 and 1.36, respectively, compared to the OA-LCNPG1 gel preparation. There were two processes in the infiltration study, including the initial burst effect followed by the gradual release of OA, which is consistent with previous in vitro release studies.

The in vitro drug release from the optimized OALCNP-G1 was best explained by Higuchi's equation, as the plots showed the highest linearity $\left(R^{2}=0.989\right)$, followed by first order $\left(R^{2}=0.924\right)$ and zero order $\left(R^{2}=\right.$ 0.887). The corresponding plot of ( $\log \%$ cumulative drug release vs log time) for the Korsmeyer-Peppas equation indicated good linearity $\left(R^{2}=0.963\right)$. The value $\mathrm{r}^{2}$ of Peppas equation indicated good linearity showing irregular (non-Fickian) diffusion which means that drug release was controlled by more than one process, and the diffusion may be controlled as well as swelling controlled release as shown in Table 7.

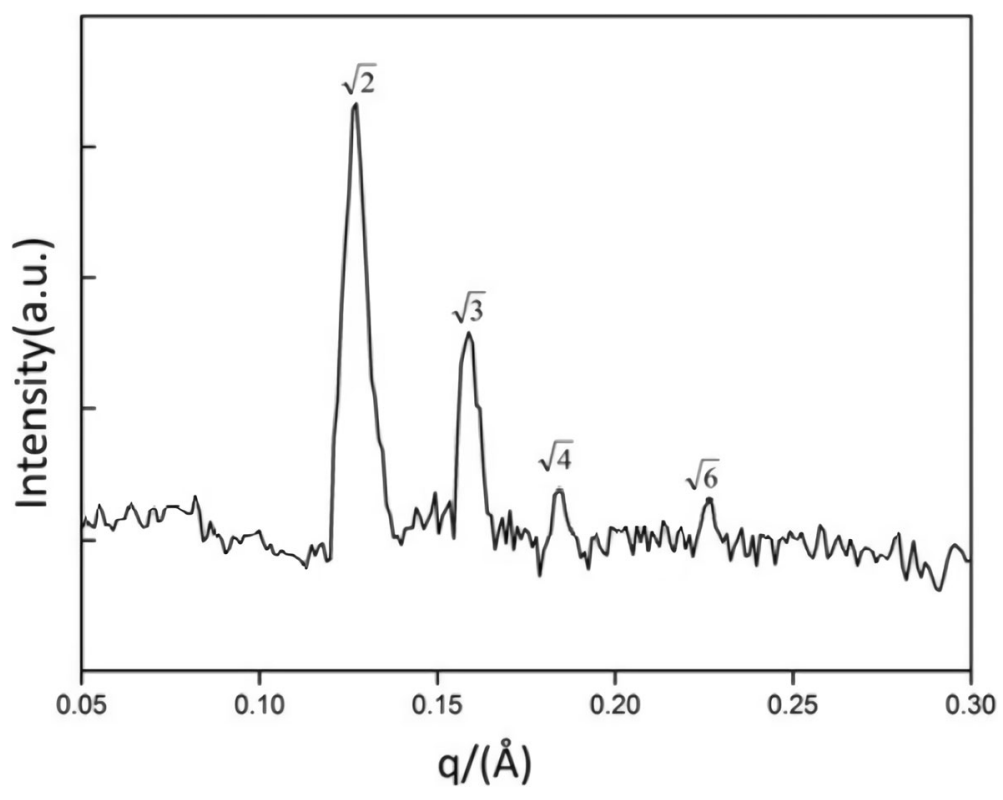

Fig. 6 Intensity versus q plot from SAXS measurement of OA-loaded LCNP 


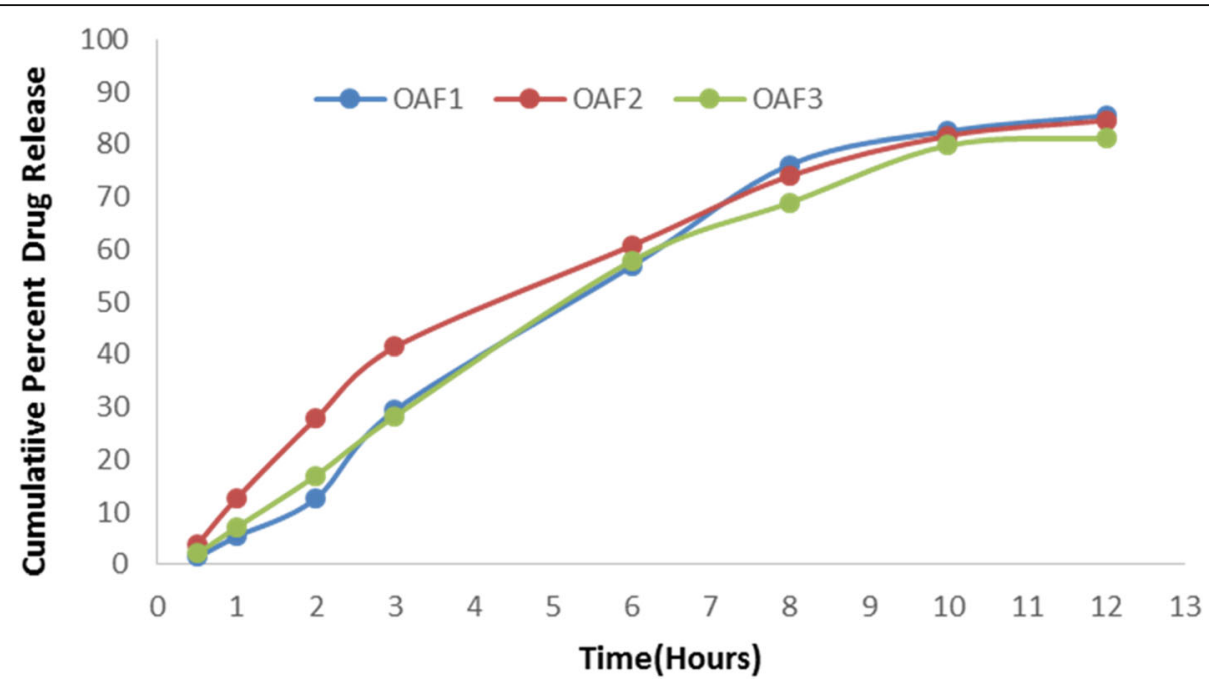

Fig. 7 - In vitro drug release profiles of OA-loaded LCNP up to $12 \mathrm{~h}$

Table 6 Characterization parameters for OA-LCNP-loaded gel

\begin{tabular}{llllll}
\hline S.No. & Formulation & pH & Viscosity(cps) & $\begin{array}{l}\text { Spreadability } \\
\left(\mathbf{g} \cdot \mathbf{c m} \cdot \mathbf{m i n}^{-1} \mathbf{)}\right.\end{array}$ & $\begin{array}{l}\text { Drug content } \\
\mathbf{( \% ~} \mathbf{w} / \mathbf{w})\end{array}$ \\
\hline 1. & OA-LCNP-G1 & 7.3 & $72,000 \sim 79,000$ & $258.98 / 2.35$ & $96.12 \pm 0.31$ \\
2. & OA-LCNP-G2 & 7.4 & $64,000 \sim 71,000$ & $241.33 / 2.43$ & $97.32 \pm 0.29$ \\
3. & OA-LCNP-G3 & 7.3 & $55,000 \sim 63,000$ & $247.91 / 2.41$ & $96.89 \pm 0.12$ \\
4. & NG & 7.4 & $67,000 \sim 73,000$ & $246.38 / 3.13$ & $97.46 \pm 0.41$ \\
\hline
\end{tabular}

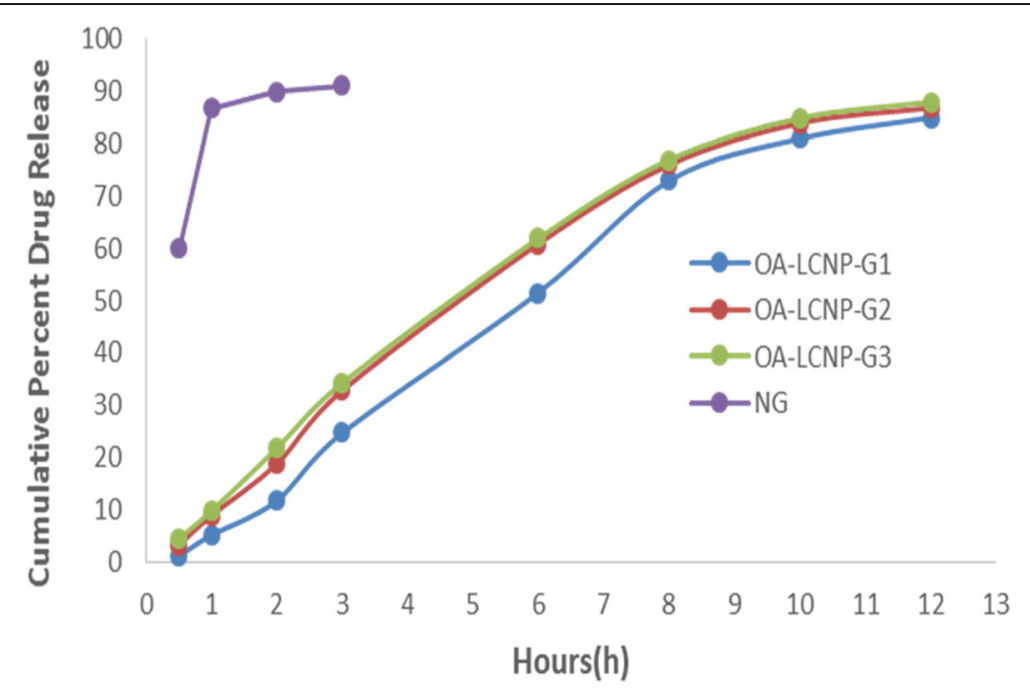

Fig. 8 In vitro drug release profiles of OA-LCNP-loaded topical gels up to $12 \mathrm{~h}$ 


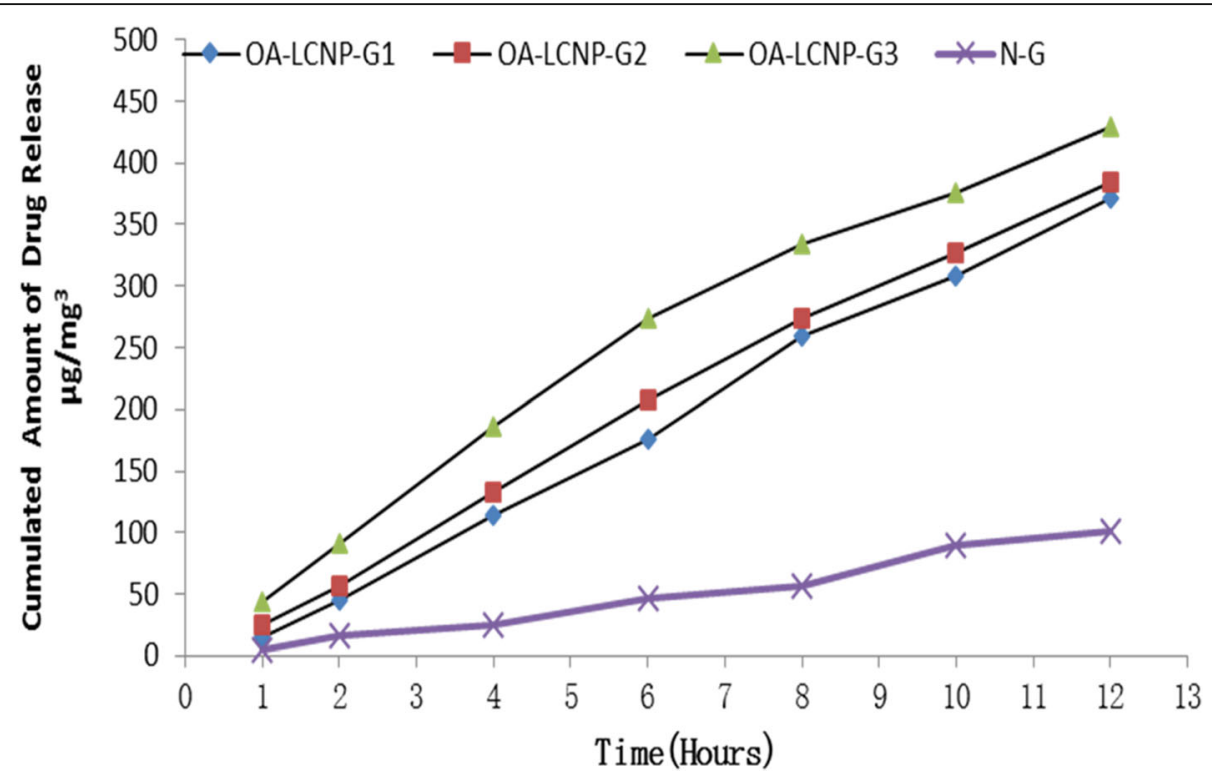

Fig. 9 Ex vivo permeation results of different OA-LCNP-loaded gel formulations

In vivo anti-inflammatory studies

The anti-inflammatory activity of the optimized preparation for OA-LCNP-loaded topical gel was evaluated using the carrageenan-induced hind paw inflammation method on Wistar rats. The percentage inhibition value of OA-LCNP gel (test) was also compared to marketed mometasone furoate gel (standard) as displayed in Table 8. OA-LCNP gel decreased the inflammation to a certain degree and also revealed a sustained effect for a prolonged period.

\section{Conclusions}

In this study, OA-loaded LCNP was prepared by the precursor method, resulting in a well-characterized OA-LCNP gel preparation. The gel was shown to be effective in a rodent carrageenan-induced hind paw inflammation with sustained efficacy after a single application, but there is still a pressing need for further in vivo studies to realize their full potential.

Table 7 Release kinetic parameters for OA-loaded LCNP gel (OA-LCNP-G2)

\begin{tabular}{|c|c|c|c|c|c|c|c|}
\hline \multicolumn{2}{|c|}{ Zero order } & \multicolumn{2}{|c|}{ First order equation } & \multicolumn{2}{|c|}{ Peppas equation } & \multicolumn{2}{|c|}{ Higuchi equation } \\
\hline$k$ & $R^{2}$ & $k$ & $R^{2}$ & $k$ & $R^{2}$ & $k$ & $R^{2}$ \\
\hline 3.124 & 0.887 & 0.0416 & 0.924 & 0.6048 & 0.963 & 18.212 & 0.989 \\
\hline
\end{tabular}

Table 8 In vivo anti-inflammatory activity: carrageenan-induced hind paw edema

\begin{tabular}{|c|c|c|c|c|c|c|c|c|}
\hline \multirow[t]{2}{*}{ Treatment } & \multirow{2}{*}{$\begin{array}{l}\text { Anti- } \\
\text { inflammatory } \\
\text { activity }\end{array}$} & \multicolumn{7}{|l|}{ Time } \\
\hline & & 0 & 1 & 2 & 4 & 8 & 12 & 24 \\
\hline Control & Edema rate & $0.00 \pm 0.02$ & $34.12 \pm 0.02$ & $43.87 \pm 0.02$ & $44.32 \pm 0.01$ & $45.87 \pm 0.02$ & $46.98 \pm 0.02$ & $40.92 \pm 0.02$ \\
\hline \multirow[t]{2}{*}{ Test } & Edema rate & $0.00 \pm 0.01$ & $32.28 \pm 0.02$ & $39.56 \pm 0.02$ & $36.81 \pm 0.02$ & $36.13 \pm 0.02$ & $35.41 \pm 0.02$ & $25.28 \pm 0.02$ \\
\hline & Inhibition rate(\%) & & 5.61 & 7.81 & 17.32 & 20.83 & 24.89 & 45.26 \\
\hline Standard & Edema rate & $0.00 \pm 0.01$ & $31.72 \pm 0.02$ & $34.11 \pm 0.02$ & $31.87 \pm 0.02$ & $26.13 \pm 0.02$ & $21.09 \pm 0.02$ & $17.42 \pm 0.02$ \\
\hline
\end{tabular}




\section{Acknowledgements}

The authors would like to express their gratitude to EditSprings (https:// www.editsprings.com/) for the expert linguistic services provided.

\section{Authors' contributions}

Z.Q.S. and S.G.P. conceptualized this study, and should be considered co-first authors. Z.Q.S. and S.G.P. ran the experiments. Z.Q.S., L.L.W.and S.L. analyzed the data and contributed to the writing of the paper. All authors read and approved the final manuscript.

\section{Funding}

This project was supported by the Natural Science Foundation from education department of Jiangsu Province, China (Grant Number: 19KJB360001) and Changzhou Science and Technology and Information Bureau (Grant Number: CJ20189003 and CJ20190011).

\section{Availability of data and materials}

All data generated or analysed during this study are included in this published article.

\section{Declarations}

\section{Ethics approval and consent to participate}

The study was carried out in compliance with the ARRIVE guidelines. The experimental protocols and treatments were all approved by Ethics Committee of Guangdong Provincial Institute of Traditional Chinese Medicine. All methods were carried out in accordance with the Guangdong Province Laboratory Animal Care and Use Guidelines.

\section{Consent for publication}

Not applicable.

\section{Competing interests}

The authors declare that they hava no competing interests.

\section{Author details}

${ }^{1}$ Changzhou Institute of Industry and Technology, No.28\#, Mingxin Road, Changzhou 213164, Jiangsu Province, China. ${ }^{2}$ Changzhou Institute of Technology, Changzhou 213022, Jiangsu Province, China. ${ }^{3}$ Key Laboratory for Soft Chemistry and Functional Materials of Ministry Education, Nanjing University of Science and Technology, Nanjing 211816, Jiangsu Province, China. ${ }^{4}$ Guangdong Provincial Institute of Traditional Chinese Medicine, Guangzhou 510095, Guangdong Province, China. ${ }^{5}$ Wuxi Hospital of Traditional Chinese Medicine, No.8\#, Zhongnan Road, Wuxi 214071, Jiangsu Province, China.

\section{Received: 18 April 2021 Accepted: 17 August 2021}

Published online: 04 September 2021

\section{References}

1. Liu J. Pharmacology of oleanolic acid and ursolic acid. J Ethnopharmacol. 1995;49(2):57-68. https://doi.org/10.1016/0378-8741(95)90032-2.

2. Dzubak P, Hajduch M, Vydra D, Hustova A, Kvasnica M, Biedermann D, et al. Pharmacological activities of natural triterpenoids and their therapeutic implications. Nat Prod Rep. 2006;23(3):394-411. https://doi.org/10.1039/b51 5312n.

3. Pollier J, Goossens A. Oleanolic acid. Phytochem. 2012;77:10-5. https://doi. org/10.1016/j.phytochem.2011.12.022.

4. Somova LI, Shode FO, Ramnanan P, Nadar A. Antihypertensive, antiatherosclerotic and antioxidant activity of triterpenoids isolated from Olea europaea, subspecies africana leaves. J Ethnopharmacol. 2003;84(2-3): 299-305. https://doi.org/10.1016/S0378-8741(02)00332-X.

5. Singh GB, Singh S, Bani S, Gupta BD, Banerjee SK. Anti-inflammatory activity of oleanolic acid in rats and mice. J Pharm Pharmacol. 1992;44(5):456-8. https://doi.org/10.1111/j.2042-7158.1992.tb03646.x.

6. Kurek A, Nadkowska P, Pliszka S, Wolska Kl. Modulation of antibiotic resistance in bacterial pathogens by oleanolic acid and ursolic acid. Phytomedicine. 2012;19(6):515-9. https://doi.org/10.1016/j.phymed.2 011.12.009.
7. Lee W, Yang EJ, Ku SK, Song KS, Bae JS. Anti-inflammatory effects of Oleanolic acid on LPS-induced inflammation. In Vitro In Vivo Inflam. 2013; 36(1):94-101. https://doi.org/10.1007/s10753-012-9523-9.

8. Bednarczyk-Cwynar B, Wachowiak N, Szulc M, et al. Strong and long-lasting Antinociceptive and anti-inflammatory conjugate of naturally occurring Oleanolic acid and aspirin. Front Pharmacol. 2016;7:1-18.

9. Dharmappa KK, Kumar RV, Nataraju A, Mohamed R, Shivaprasad HV, Vishwanath BS. Anti-inflammatory activity of oleanolic acid by inhibition of secretory phospholipase A2. Planta Med. 2009;75(3):211-5. https://doi.org/1 0.1055/s-0028-1088374.

10. Kashyap D, Sharma A, Tuli HS, Punia S, Sharma AK. Ursolic acid and Oleanolic acid: Pentacyclic Terpenoids with promising anti-inflammatory activities. Recent Patents Inflamm Allergy Drug Discov. 2016;10(1):21-33. https://doi.org/10.2174/1872213X10666160711143904.

11. Kurek A, Nadkowska R, Pliszka S, Wolska Kl. Modulation of antibiotic resistance in bacterial pathogens by oleanolic acid and ursolic acid. Phytomedicine. 2012; 19(6):515-9. https://doi.org/10.1016/.jphymed.2011.12.009.

12. Seung-Hyung $\mathrm{K}$, Jung-hee $H$, Young-Cheol L. Oleanolic acid suppresses ovalbumin-induced airway inflammation and Th2-mediated allergic asthma by modulating the transcription factors Tbet, GATA-3,RORt and Foxp3 in asthmatic mice. Int Immunopharmacol. 2012;18(2):311-24.

13. Lim HJ, Jang HJ, Kim MH, Lee S, Lee SW, Lee SJ, et al. Oleanolic acid acetate exerts anti-inflammatory activity via IKKa/ $\beta$ suppression in TLR3-mediated NF-KB activation. Molecules. 2019;24(21):4002. https://doi.org/10.3390/ molecules 24214002

14. Liu J. Oleanolic acid and ursolic acid: research perspectives. J Ethnopharmacol. 2005;100(1-2):92-4. https://doi.org/10.1016/j.jep.2005.05.024.

15. Jeong DW, Kim YH, Kim HH, Ji HY, Yoo SD, Choi WR, et al. Dose-linear pharmacokinetics of oleanolic acid after intravenous and oral administration in rats. Biopharm Drug Dispos. 2007;28(2):51-7. https:// doi.org/10.1002/bdd.530.

16. Gao D, Tang S, Tong Q. Oleanolic acid liposomes with polyethylene glycol modification: promising antitumor drug delivery. Int J Nanomedicne. 2012;7: $3517-26$.

17. Tong HH, Du Z, Wang GN, Chan HM, Chang Q, Lai LC, et al. Spray freeze drying with polyvinylpyrrolidone and sodium caprate for improved dissolution and oral bioavailability of oleanolic acid, a BCS Class IV compound. Int J Pharm. 2011;404(1-2):148-58. https://doi.org/10.1016/j.jpha rm.2010.11.027.

18. Chen $Y$, Liu J, Yang $X$, Zhao $X, X u H$. Oleanolic acid nanosuspensions: preparation, in-vitro characterization and enhanced hepatoprotective effect. J Pharm Pharmacol. 2005;57:259-642. https://doi.org/10.1211/ 0022357055407.

19. Jiang $Q$, Yang $X, D u P$, Zhang $H$, Zhang $T$. Dual strategies to improve oral bioavailability of oleanolic acid: Enhancing water-solubility, permeability and inhibiting cytochrome P450 isozymes. Eur J Pharm Biopharm. 2016;99:65-72 https://doi.org/10.1016/j.ejpb.2015.11.013.

20. Esposito E, Cortesi R, Drechsler M, Paccamiccio L, Mariani P, Contado C, et al. Cubosome dispersions as delivery systems for percutaneous administration of indomethacin. Pharm Res. 2005;22(12):2163-73. https://doi. org/10.1007/s11095-005-8176-x.

21. Gan L, Han S, Shen J, Zhu J, Zhu C, Zhang X, et al. Self-assembled liquid crystalline nanoparticles as a novel ophthalmic delivery system for dexamethasone: improving preocular retention and ocular bioavailability. Int J Pharm. 2010;396(1-2):179-87. https://doi.org/10.1016/ j.jpharm.2010.06.015.

22. Rizwan SB, Assmus D, Boehnke A, Hanley T, Boyd BJ, Rades T, et al. Preparation of phytantriol cubosomes by solvent precursor dilution for the delivery of protein vaccines. Eur J Pharm Biopharm. 2011;79(1):15-22. https://doi.org/10.1016/j.ejpb.2010.12.034.

23. Yang Z, Tan Y, Chen M, Dian L, Shan Z, Peng X, et al. Development of amphotericin B-loaded cubosomes through the SolEmuls technology for enhancing the oral bioavailability. AAPS Pharm Sci Tech. 2012;13(4):1483-91. https://doi.org/10.1208/s12249-012-9876-2.

24. Mei $L$, Xie $Y$, Jing H, Huang $Y$, Chen J, Ran $H$, Pan X, Wu C. A novel design for stable self-assembly cubosome precursormicroparticles enhancing dissolution of insoluble drugs. Drug Dev Ind Pharm. 2017;43(8):1239-43. https://doi.org/10.1080/03639045.2017.1304958.

25. Deshpande S, Singh N. Influence of Cubosome surface architecture on its cellular uptake mechanism. Langmuir. 2017;33(14):3509-16. https://doi.org/1 0.1021/acs.langmuir.6b04423. 
26. Tu YS, Fu JW, Sun DM, Zhang JJ, Yao N, Huang DE, et al. Preparation, characterisation and evaluation of curcumin with piperine-loaded cubosome nanoparticles. J Microencapsul. 2014;31(6):551-9. https://doi. org/10.3109/02652048.2014.885607.

27. Spicer PT, Hayden KL, Lynch ML, Ofori-Boateng A, Burns JL. Novel process for producing cubic liquid crystalline nanoparticles (cubosomes). Langmuir. 2001;17(19):5748-56. https://doi.org/10.1021/la010161w.

28. Sanad RA, Abdelmalak NS, Elbayoomy TS, Badawi AA. Formulation of a novel oxybenzone-loaded nanostructured lipid carriers (NLCS). AAPS PharmSciTech. 2010;11(4):1684-94. https://doi.org/10.1208/s12249-0109553-2.

29. Nagaich U, Gulati N. Nanostructured lipid carriers (NLC) based controlled release topical gel of clobetasol propionate: design and in vivo characterization. Drug Deliv Transl Res. 2016;6(3):289-98. https://doi.org/10.1 007/s13346-016-0291-1.

30. Thatipamula RP, Palem CR, Gannu R, Mudragada S, Yamsani M. Formulation and in vitro characterization of domperidone loaded solid lipid nanoparticles and nanostructured lipid carriers. Daru. 2011;19(1):23-32.

31. Achdout H, Lustig S, Israely T, Erez N, Politi B, Tamir H, et al. Induction, treatment and prevention of eczema vaccinatum in atopic dermatitis mouse models. Vaccine. 2017;35(33):4245-54. https://doi.org/10.1016/j.va ccine.2017.06.014.

32. Sagalowicz L, Leser ME, Watzke HJ, Michel M. Monoglyceride self-assembly structures as delivery vehicles. Trends Food Sci Technol. 2006;17(5):204-14. https://doi.org/10.1016/j.tifs.2005.12.012.

33. Barauskas J, Landh T. Phase behavior of the Phytantriol/water system. Langmuir. 2003;19(23):9562-5. https://doi.org/10.1021/la0350812.

34. Landh T. Phase behavior in the system pine needle oil monoglycerides poloxamer 407-water at 20.Degree. J Phys Chem. 1994;98:8453-6734. https://doi.org/10.1021/j100085a028.

35. Ata S, Rasool A, Islam A, Bibi I, Rizwan M, Azeem MK, et al. Loading of Cefixime to $\mathrm{pH}$ sensitive chitosan based hydrogel and investigation of controlled release kinetics. Int J Biol Macromol. 2020;155:1236-44. https:// doi.org/10.1016/j.ijbiomac.2019.11.091.

36. Li B, Ge ZQ. Nanostructured lipid carriers improve skin permeation and chemical stability of idebenone. AAPS PharmSciTech. 2012;13(1):276-83. https://doi.org/10.1208/s12249-011-9746-3

\section{Publisher's Note}

Springer Nature remains neutral with regard to jurisdictional claims in published maps and institutional affiliations.

Ready to submit your research? Choose BMC and benefit from:

- fast, convenient online submission

- thorough peer review by experienced researchers in your field

- rapid publication on acceptance

- support for research data, including large and complex data types

- gold Open Access which fosters wider collaboration and increased citations

- maximum visibility for your research: over $100 \mathrm{M}$ website views per year

At $\mathrm{BMC}$, research is always in progress.

Learn more biomedcentral.com/submissions 